\title{
Pascal CYR, Waterloo : origines et enjeux
}

Paris, L'Harmattan, coll. Historiques, 2011, 439 p., ISBN

978-2-296-54244-0, $39 €$.

\section{Annie Crépin}

\section{OpenEdition}

\section{Journals}

Édition électronique

URL : https://journals.openedition.org/ahrf/12913

DOI : $10.4000 /$ ahrf.12913

ISSN : 1952-403X

Éditeur :

Armand Colin, Société des études robespierristes

Édition imprimée

Date de publication : 1 septembre 2013

Pagination : 226-228

ISBN : 9782200928261

ISSN : 0003-4436

Référence électronique

Annie Crépin, «Pascal cyr, Waterloo : origines et enjeux », Annales historiques de la Révolution française [En ligne], 373 | juillet-septembre 2013, mis en ligne le 03 octobre 2013, consulté le 01 juillet 2021. URL : http://journals.openedition.org/ahrf/12913; DOI : https://doi.org/10.4000/ahrf.12913

Ce document a été généré automatiquement le 1 juillet 2021.

Tous droits réservés 


\section{Pascal CYR, Waterloo : origines et enjeux}

Paris, L'Harmattan, coll. Historiques, 2011, 439 p., ISBN

978-2-296-54244-0, $39 €$.

Annie Crépin

\section{RÉFÉRENCE}

Pascal CYR, Waterloo : origines et enjeux. Paris, L'Harmattan, coll. Historiques, 2011, 439 p., ISBN 978-2-296-54244-0, $39 €$.

1 Pascal Cyr, docteur de l'Université de Montréal, a souhaité mettre le résultat de ses recherches à la disposition d'un large public. Dans l'introduction de son ouvrage, il déplore que l'histoire bataille, délaissée par les universitaires selon lui, soit " accaparée» par les historiens amateurs qui reproduisent inlassablement les mêmes thèses et n'utilisent guère les sources de première main. Pour remédier à ces carences, il relit ces sources et replace la bataille de Waterloo dans un large contexte, qu'il n'hésite pas à faire remonter parfois jusqu'à l'année 1805. On pourra objecter à l'auteur que l'histoire bataille a connu une véritable révolution, illustrée précisément dans le cas de Waterloo et selon une approche différente, par les travaux de Jean-Marc Largeaud et de John Keegan, qu'il cite dans sa bibliographie mais peu au cours de son étude. Mais il déclare peu concluant l'exercice de ce dernier à la fin de son ouvrage dans laquelle il se livre à une relecture critique de l'historiographie de la bataille - qu'il aurait été plus judicieux de placer au commencement du livre. Et au final son analyse aboutit, non pas à une œuvre relevant de la nouvelle histoire-bataille mais - et on ne saurait lui en faire le reproche - à un récit extrêmement classique du combat.

2 Par ailleurs la perspective de vulgarisation très louable en soi adoptée par Pascal Cyr le conduit à des raccourcis audacieux et même hasardeux, à des formulations approximatives ou qui mériteraient d'être davantage nuancées, voire à une présentation quelque peu simplificatrice qui aggrave la confusion plus qu'elle ne la 
dissipe et amène parfois à des contre-sens. Citons par exemple les "privilèges » (p. 17) acquis sous la Révolution et l'Empire que la déclaration de Saint-Ouen promet de conserver, l'Église « annexée » par Bonaparte depuis la promulgation du Concordat (p. 86), les conditions de vie du bas-clergé qui n'auraient pas été améliorées par le Concordat, certains curés ayant «tôt fait de se tourner vers la mendicité » (p. 89), le fait que l'Acte additionnel aux Constitutions de l'Empire, en affirmant la liberté de culte, aurait «apporté une sévère entorse au Concordat qui [...] stipulait que la religion catholique était prédominante» (p. 139). Citons encore " des senatus-consultes qui lui [l'Empereur] permettent de lever des soldats sans passer par l'approbation du Sénat " (p. 74) (cette approbation existe même si, émanant d'un Sénat docile, elle est toute formelle et acquise d'avance !). À propos du suffrage universel, l'auteur affirme que la constitution de l'an VIII « a cette particularité de faire disparaitre le suffrage universel parce qu'elle se limite [ ...] à ceux qui paient des impôts, donc aux notables » (p. 71). Présentation contestable qui n'évoque pas l'histoire antérieure, notamment celle de la constitution de l'an III, et qui confond le fait que le suffrage universel soit vidé de son sens avec sa disparition!

3 Huit chapitres composent l'ouvrage qui comporte 439 pages et la moitié d'entre eux est consacrée au contexte politique, diplomatique et militaire, économique et financier dans lequel s'inscrit la défaite. Autant de vastes synthèses qui structurent une grande masse d'informations et qui tendent toutes à montrer que Napoléon est acculé à remporter une victoire décisive, ce qui n'est pas sans conséquences sur la stratégie et même sur la tactique qu'il adopte lors des combats qui précèdent Waterloo et à Waterloo même. Plusieurs de ces chapitres contiennent une comparaison avec la situation à la veille d'Austerlitz, autre bataille décisive au cours de cette année 1805 en laquelle l'auteur voit - il le dit dès l'introduction - le début du déclin de l'empire napoléonien, hypothèse plus affirmée que démontrée au cours de l'ouvrage.

4 Le premier de ces chapitres évoque les enjeux du Congrès de Vienne ainsi que l'état des rapports de force en Europe et, avec bonheur, le rôle que joue alors Talleyrand; le suivant analyse l'inertie, quand ce n'est pas de la résistance passive, dont font preuve à tous les échelons les diverses instances administratives, qui concorde avec l'attentisme des notables et la méfiance des libéraux évoqués dans le chapitre quatrième et les mouvements d'opposition royaliste analysés dans le chapitre troisième. Pascal Cyr montre fort bien qu'en général ceux-ci échouent, qu'ils ne reposent pas sur un soutien populaire à la cause des Bourbons et qu'ils n'entraînent pas non plus un soulèvement de grande ampleur. Mais celui de l'Ouest paraît suffisamment inquiétant à Napoléon pour qu'il se sente obligé de soustraire 10000 hommes à l'Armée du Nord.

Les quatre chapitres suivants sont consacrés à la campagne qui précède Waterloo et à la bataille elle-même. Ce sont les meilleurs de l'ouvrage, même s'ils se développent dans les cadres d'une narration très classique. Chacun de ces chapitres se termine de façon très judicieuse par un résumé bien utile pour le lecteur. On regrettera par contre qu'il y ait fort peu de cartes et que celles qui existent ne soient pas très lisibles. Le cinquième chapitre analyse avec précision la préparation de la campagne notamment sous l'angle de ses incidences financières et pratiques. Il expose le cercle vicieux dans lequel est enfermé l'Empereur: obligé de remporter une victoire décisive pour se rallier les milieux financiers, il a aussi impérativement besoin d'argent pour mener à bien sa campagne. Or l'auteur a déjà montré précédemment que les sources de financement se tarissent et que les fournisseurs ne font plus crédit à l'Empire. La guerre va donc payer 
la guerre. Toutefois, dans un premier temps et grâce à un certain nombre d'expédients qui creusent la dette de l'État, il réussit à équiper convenablement l'armée qui entre en campagne.

6 Il lui faut faire vite pour toutes les raisons évoquées plus haut et qui ne sont pas d'ordre purement militaire; d'où le choix d'une stratégie offensive (contre l'avis de Carnot qui aurait préféré qu'on s'appuie sur les places-fortes, plan dont Pascal Cyr remet en cause la pertinence) et le dessein de combattre séparément Prussiens et Anglais afin de leur ôter l'avantage que leur donnait leur supériorité numérique, une fois réunis, et par conséquent le choix d'une attaque du dispositif ennemi en son centre. Mais ce qui réussissait quand la Grande Armée le mettait en œuvre va être pratiqué par l'Armée du Nord dont l'auteur a montré qu'elle n'était pas à la hauteur de sa devancière.

7 Finalement le plan échoue, Pascal Cyr le montre dans l'étude minutieuse des combats du 16 juin, ceux de Ligny et des Quatre-Bras où sont « ratés » successivement Blücher et Wellington. L'auteur a soin de démêler l'écheveau des responsabilités et évite de faire de tel ou tel un bouc-émissaire, y compris l'empereur dont il ne cèle cependant pas les erreurs d'appréciation sur le terrain. C'est tout « un ensemble d'événements mineurs en soi » (p. 263) qui entraîne la défaite finale, y compris la malchance qu'en d'autres temps le général de l'Armée d'Italie aurait peut-être surmontée.

8 La même volonté d'éviter le morceau de bravoure et de s'en tenir à l'exposé des faits, non sans manifester une distance critique envers les interprétations précédentes, prévaut pour Waterloo. Pascal Cyr montre que le retour en arrière semblait être la seule alternative raisonnable à la bataille mais qu'un tel retour était impossible pour les raisons politiques exposées plus haut. La politique intérieure a donc eu un retentissement sur la tactique adoptée pendant le combat du 18 juin, manœuvre sur position centrale, désormais très risquée, voire sur l'impossibilité pour l'empereur, condamné à la victoire, d'ordonner une retraite quand il en était encore temps. Il est désormais condamné à l'abdication.

9 On ne peut s'empêcher de penser qu'il aurait été préférable que l'ouvrage soit davantage centré sur Waterloo et plus concis quant au contexte, même s'il était bon de ne pas s'en abstraire et de montrer que l'histoire militaire est aussi une histoire politique. 